\title{
EMBRYONIC DEVELOPMENT OF IDENTIFIED NEURONS: SEGMENT- SPECIFIC DIFFERENCES IN THE H CELL HOMOLOGUES ${ }^{1}$
}

\author{
MICHAEL BATE, ${ }^{*}$ COREY S. GOODMAN, $\ddagger \S$ AND NICHOLAS C. SPITZER§
}

*Max-Planck-Institut für Virusforschung, Tübingen, F.D.R.; $\ddagger$ Department of Biological Sciences, Stanford University, Stanford, California 94305; and \$ Department of Biology, B-022, University of California, San Diego, La Jolla, California 92093

\begin{abstract}
Although all of the segmental ganglia of the grasshopper come from identical sets of embryonic precursor cells, there are striking differences in the number and properties of the neurons in the mature ganglia. In every segment, midline precursor 3 (MP3) divides once and gives rise to two progeny. We describe the fate of the two MP3 progeny in different segments. The morphology, physiology, and survival of the MP3 progeny depend upon their segmental position in the embryo.

In the meso- and metathoracic segments (T2 and T3), one of the two progeny of MP3 transforms into the $\mathrm{H}$ cell. In many of the abdominal segments, beginning with the fourth (A4), both MP3 progeny die. A gradient of transformation and cell death is seen in the intervening first three abdominal segments (A1 to A3). From A1 to A3, the MP3 progeny acquire fewer of the morphological phenotypes of the $\mathrm{H}$ cell. This is paralleled by the partial acquisition of the physiological phenotypes of the $H$ cell. Thus, cells that are the progeny of the same precursor cell in different segments can either live or die and, if they survive, can develop different morphological and physiological properties.
\end{abstract}

Many metazoan animals have a metameric organization and generate a segmentally repeating central nervous system with regional specializations. Two possible ways of constructing such a nervous system are to (i) build each segment independently from the outset or (ii) build equivalent segments and then sculpt them to meet individual requirements by cell death and segment-specific differences in cellular differentiation. The grasshopper is a segmental animal whose neuromeres are specialized for particular functions. The segmental ganglia in the thorax and abdomen come from identical sets of embryonic precursor cells (Bate, 1976; Bate and Grunewald, 1980), yet there are striking differences in the number and properties of the neurons in the mature ganglia. How do these segmental differences arise? Can we detect segment-specific differences in developmentally homologous neurons?

In the previous paper (Goodman et al., 1981), we described a precursor cell, called midline precursor 3 (MP3), and its two progeny; these two cells are repeated in every segment. In this paper, we examine the fate of the two MP3 progeny in different segments of the embryonic grasshopper and describe the segmental differences in their survival and cell death. Furthermore, we

\footnotetext{
${ }^{1}$ This work was supported by Deutsche Forschungsgemeinschaft to C. M. B., the Helen Hay Whitney Foundation and a National Science Foundation grant to C. S. G., and National Institutes of Health and National Science Foundation grants to N. C. S. We thank E. B. Grunewald, J. Coulombe, and K. Ridge for technical assistance.
}

show that in those segments in which these cells survive, they develop segment-specific differences in their morphology and physiology. Some of these results have previously been reported (Goodman et al., 1979a; Spitzer et al., 1979).

\section{Methods}

All methods used here have been described in previous papers (Bate, 1976; Bate and Grunewald, 1980; Goodman and Spitzer, 1979; Goodman et al., 1979b; Goodman et al., 1981). We observe the death and disappearance of neurons in two ways: $(i)$ observation of living embryos at successive stages viewed with interference contrast optics and (ii) observation of fixed embryos reconstructed from serial sections.

\section{Results \\ Transformation of the $H$ cell}

The $\mathrm{H}$ cell is transformed during the embryonic development of the metathoracic (T3) segment (Goodman et al., 1981). This transformation involves the loss of its initial process and the acquisition of new morphological and physiological properties. The $\mathrm{H}$ cell originates from the single cell division of midline precursor 3 (MP3) on day 5 in the thoracic segments and slightly later, more posteriorly. The two MP3 progeny are initially bilaterally symmetric; each cell has a single contralateral process which extends anteriorly in one of the longitudinal fiber tracts. These processes are among the earliest in these 
tracts. Several days after the initial process extension, one of the two cells extends a new growth cone which bifurcates in the posterior commissure and then each axon bifurcates at the longitudinal fiber tracts. The cell transiently has its original unilateral process and its new set of axons which give it its characteristic " $\mathrm{H}$ " morphology. Shortly thereafter, the original process disappears and the $\mathrm{H}$ cell is left with only its new axons extending anteriorly and posteriorly in both longitudinal fiber tracts. Thus, it first appears as one of a pair of central pioneer fibers and then transforms into an unpaired identified neuron. Its sibling (sib) often dies, although when it persists, it retains only its original form.

\section{Segment-specific differences in the MP3 progeny}

Cell death. In many of the abdominal segments, the MP3 progeny die about half way through embryogenesis around day 10 . In the fourth through sixth abdominal segments (A4 to A6), the MP3 progeny die in more than $90 \%$ of the embryos (Fig. 1). In T3, both cells often survive, with one transforming into the $\mathrm{H}$ cell and the other remaining morphologically unchanged; in about $20 \%$ of the embryos, however, the $\mathrm{H}$ cell sib dies. A similar pattern of survival and transformation is observed in $\mathrm{T} 2$, with the exception that the $\mathrm{H}$ cell sib dies in about $40 \%$ of the embryos. A gradient of cell death is seen in the first three abdominal ganglia (A1 to A3) at the boundary between thorax and abdomen. The typical pattern of survival is two cells in A1, one cell in A2, and no cells in A3. A great deal of variability is observed, however (Fig. 1). For example, in about $10 \%$ of the embryos, only one of the two MP3 progeny survives in A1 (Fig. 2). Cell death in the MP3 progeny occurs by day 12 ; if the cells survive past this point, then they survive through hatching on day 20.

Morphological transformation. In T2 and T3, one of the two MP3 progeny fully transforms into the $\mathrm{H}$ cell.

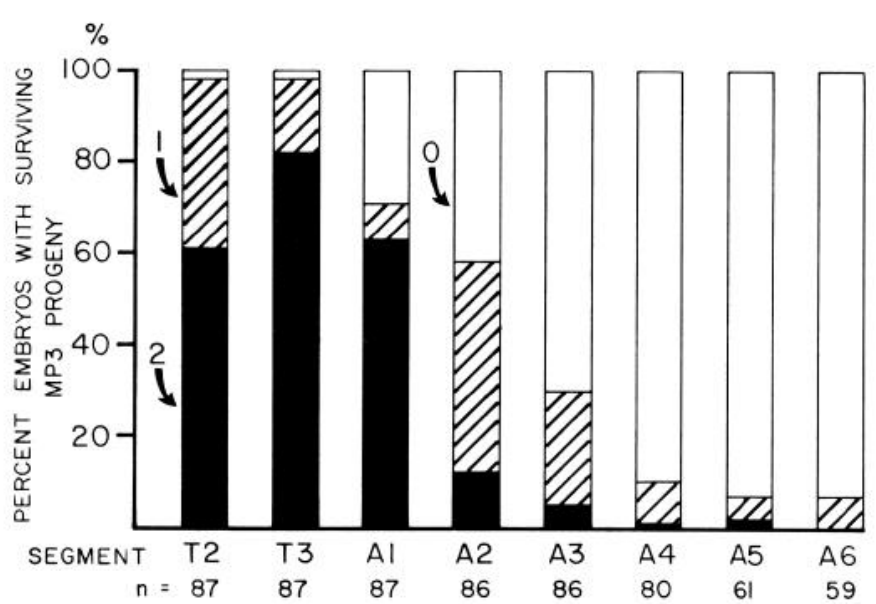

Figure 1. The survival and cell death of the two MP3 progeny in different segments of grasshopper embryos in dissected preparations examined at day 12 to day 16 . The percentage of embryos with two (solid bar), one (hatched bar), or no (open bar) MP3 progeny surviving per segment is scored for the mesothoracic and metathoracic (T2 and T3) and the first six abdominal ganglia (A1 to A6). The number of embryos examined is indicated $(n)$.

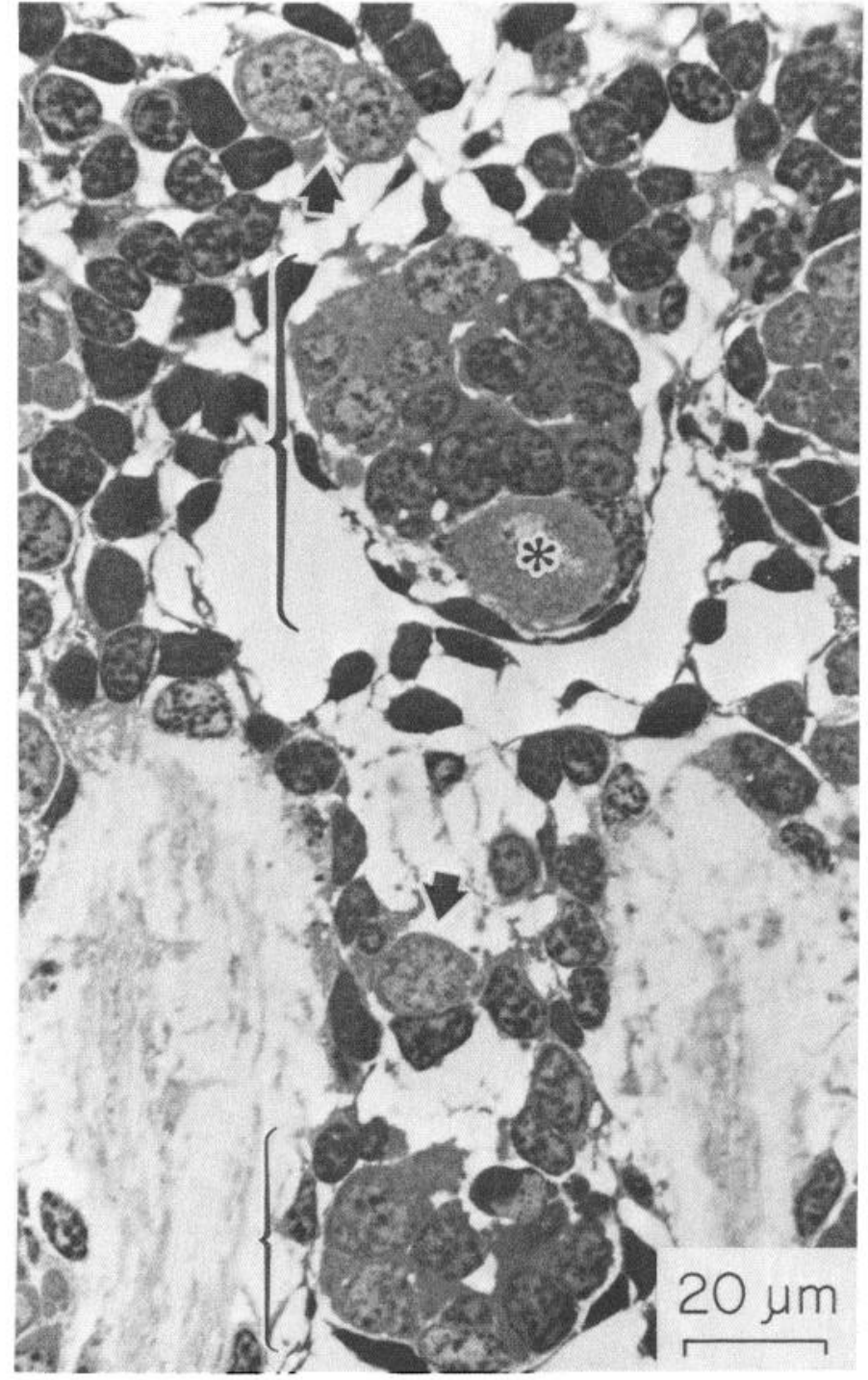

Figure 2. Photomicrograph of a horizontal section through the dorsal surface of the metathoracic (T3) and first abdominal (A1) ganglia in a day 11 grasshopper embryo. Anterior at top. The median neuroblast $(\mathrm{MNB} ; *)$ in $\mathrm{T} 3$ and some of its progeny are indicated by the bracket at the top. Both of the progeny of MP3 have survived in T3 (arrow); the larger of the two cells is the $\mathrm{H}$ cell. Some of the progeny of the MNB in Al are indicated by the bracket at the bottom. The sole survivor of the MP3 progeny in Al is indicated by the arrow.

This includes the loss of its initial process and the acquisition of new axons which extend anteriorly and posteriorly in both longitudinal fiber tracts. In those cells which survive in $\mathrm{A} 1$ to $\mathrm{A} 3$, a gradient of morphological transformation is observed (Fig. 3). As their mature form from A1 to A3, the MP3 progeny acquire fewer of the morphological phenotypes of the $\mathrm{H}$ cell. They lose their original unilateral process less often, and they extend fewer of the new axons. Examples of their morphology are shown in Figure 3; the pattern is schematically illustrated in Figure 4. In A1, usually both of the cells survive, with one transforming and the other not. The cell that transforms in A1 loses its original process and acquires new axons which only extend posteriorly in the longitu- 


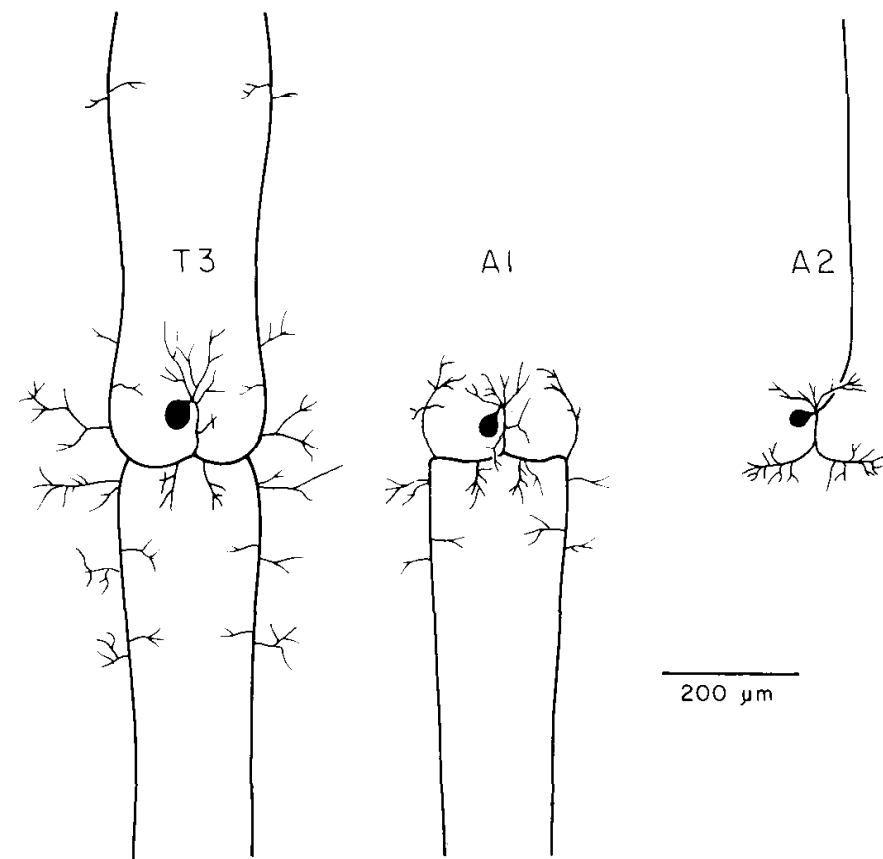

Figure 3. Segmental differences in the morphological transformation of the MP3 progeny in those cases in which they survive. These are camera lucida drawings of cells filled with Lucifer Yellow in days 13 to 16 embryos. The complete transformation of the $\mathrm{H}$ cell is seen in the metathoracic (T3) segment. The posterior portion of the transformation is seen in A1. Only short central branches appear in A2; this cell did not lose its original process.
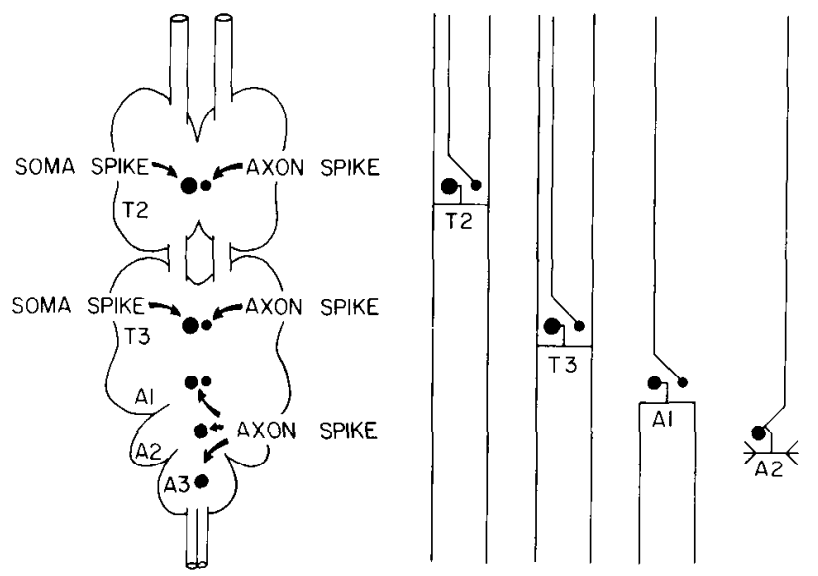

Figure 4. Segmental differences in the physiological and morphological properties of the MP3 progeny in segments T2, T3, and A1 to A3, assayed in days 12 to 16 embryos. The schematic diagrams show one common pattern in which two cells survive in $\mathrm{T} 2, \mathrm{~T} 3$, and $\mathrm{A} 1$; one cell in $\mathrm{A} 2$ and $\mathrm{A} 3$; and no cells in A4 to A6. The acquisition of only a fraction of the morphological properties is paralleled by the partial acquisition of the physiological properties; the progeny of MP3 in A1 to A3 develop excitable axons but do not develop overshooting soma spikes.

dinal fiber tracts. In A2, the one surviving cell does not lose its original process and grows a new process which bifurcates and branches bilaterally in the posterior commissure. In those embryos in which one cell survives in
A3, it also retains its original process, and grows a new process which only branches in the median fiber tract (Fig. 4).

Physiological transformation. In $\mathrm{T} 3$, the $\mathrm{H}$ cell acquires the ability to generate $\mathrm{Na}^{+}$-dependent action potentials in its axons and shortly thereafter to generate $\left(\mathrm{Na}^{+}-\mathrm{Ca}^{2+}\right)$-dependent action potentials in its soma (Goodman and Spitzer, 1980a, b; Goodman et al., 1981). The $\mathrm{H}$ cell sib, when it survives, only acquires the ability to generate action potentials in its axon. This same pattern of physiological transformation is seen in T2 (Fig. 4). However, in those MP3 progeny which survive in A1 to $\mathrm{A} 3$, including those cells which partially transform their morphology, they only acquire the ability to produce axon spikes $\left(\mathrm{Na}^{+}\right.$-dependent) and do not produce overshooting soma spikes (Figs. 4 and 5). Furthermore, the addition of tetraethylammonium (TEA) does not reveal any excitable response in their somata. Thus, we have no evidence for the presence of either $\mathrm{Na}^{+}$or $\mathrm{Ca}^{2+}$ channels in their somata membrane. It appears that the partial acquisition of only a fraction of the morphological phenotypes of the $\mathrm{H}$ cell by the MP3 progeny in A1 to A3 is paralleled by the partial acquisition of the physiological phenotypes.

In $\mathrm{T} 3$, the $\mathrm{H}$ cell and the $\mathrm{H}$ cell sib remain electrically coupled during the course of the transformation; uncoupling is a late event during this secondary differentiation. Similarly, in A1, the two progeny of MP3 remain electrically coupled while one of them partially transforms and the other does not (Fig. 5).

\section{Discussion}

The thoracic and abdominal ganglia arise from identical sets of embryonic precursors: 61 neuroblasts (NBs) (Bate, 1976) and seven midline precursors (MPs) (Bate and Grunewald, 1980). The ganglia differ dramatically in the peripheral targets that they innervate; for example, the thoracic segments have limbs and wings, whereas the abdominal segments do not. Concurrent with these segmental differences are the striking differences in the number and properties of the neurons in the mature ganglia; for example, the thoracic ganglia each have $\sim 3000$ neurons and the abdominal ganglia each have $\sim 500$ neurons.

We have shown that cells that are the progeny of the same precursor cell in different segments can develop different morphological and physiological properties and also can have different probabilities of survival and cell death. In the T2 and T3 ganglia, one of the two progeny of MP3 transforms into the $\mathrm{H}$ cell and develops proper-

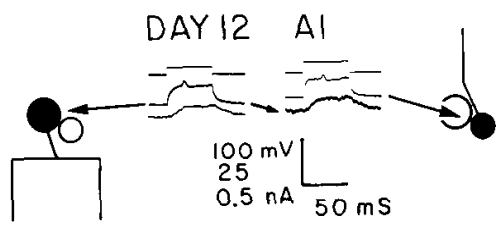

Figure 5. Electrical coupling of MP3 progeny in A1 segment during the time of the partial transformation of one of the two cells. Note that both cells acquire axon spikes but not overshooting soma spikes. In the left record, current is injected into the left cell; in the right record, it is injected into the right cell. 
ties similar to the first progeny of the median neuroblast (MNB) that are located nearby (Goodman et al., 1981). However, there are striking differences from one segmental ganglion to the next along the embryo in at least three properties. The morphology, physiology, and survival of the MP3 progeny depend upon their segmental position in the embryo. These observations raise the issue of the relative contribution of lineage and position to the differentiation of these cells.

In another insect, Drosophila, it is known that epidermal cells inherit a commitment to a particular segment; this commitment is acquired by their predecessors at an earlier stage of development (e.g., Morata and Lawrence, 1977). One possibility is that, in a similar way, precursor cells for the central nervous system in different segments could acquire segmental commitments and transmit these segmental specificities to their neuronal descendants. Such an inherited segmental commitment could account for the segment-specific differences in homologous neurons which we observe. However, at present, we have no evidence for this point of view.

Alternatively, it is possible that the precursor cells for the central nervous system and their neuronal descendants are initially segmentally uncommitted. This point of view is consistent with the reiterated pattern and number of precursor cells from segment to segment. The obvious differences in the cellular environment of different segments, particularly in the epidermal cells and underlying mesodermal cells, might then impart segment-specific differences to the otherwise segmentally uncommitted neuronal progeny. This second possibility is particularly relevant to the nervous system where the extent of cellular interactions during development is very great.

Thus, we do not know yet whether the segment-specific differences in the progeny of MP3 are generated by their segmental environment or by some inherited commitment. However, the observation that the homologous progeny of identified precursors in different segments can develop in different ways indicates how a segmentally specialized nervous system can develop from a single segmental plan.

\section{References}

Bate, C. M. (1976) Embryogenesis of an insect nervous system. I. A map of the thoracic and abdominal neuroblasts in Locusta migratoria. J. Embryol. Exp. Morphol. 35: 107-123.

Bate, C. M., and E. B. Grunewald (1980) Embryogenesis of an insect nervous system. II. A second class of neuronal precursor cells and the origin of the intersegmental connectives. J. Embryol. Exp. Morphol., in press.

Goodman, C. S., and N. C. Spitzer (1979) Embryonic development of identified neurons: Differentiation from neuroblast to neuron. Nature 280: 208-214.

Goodman, C. S., and N. C. Spitzer (1980a) The mature electrical properties of identified neurons in grasshopper embryos. J. Physiol. (Lond.), in press.

Goodman, C. S., and N. C. Spitzer (1980b) The development of electrical properties of identified neurons in grasshopper embryos. J. Physiol. (Lond.), in press.

Goodman, C. S., M. Bate, and N. C. Spitzer (1979a) Origin, transformation, and death of neurons from an identified precursor during grasshopper embryogenesis. Soc. Neurosci. Abstr. 5: 161 .

Goodman, C. S., M. O'Shea, R. McCaman, and N. C. Spitzer (1979b) Embryonic development of identified neurons: Temporal pattern of morphological and biochemical differentiation. Science 204: 219-222.

Goodman, C. S., M. Bate, and N. C. Spitzer (1981) Embryonic development of identified neurons: Origin and transformation of the H cell. J. Neurosci. 1: 94-102.

Morata, G., and P. A. Lawrence (1977) Homeotic genes, compartments and cell determination in Drosophila. Nature 265: 211-216.

Spitzer, N. C., M. Bate, and C. S. Goodman (1979) Physiological development and segmental differences of neurons from an identified precursor during grasshopper embryogenesis. Soc. Neurosci. Abstr. 5: 181. 